\title{
Estado actual y desafío del uso de TIC en la enseñanza de japonés como lengua extranjera
}

\author{
Kyoko Ito-Morales. Universidad de Granada \\ Recepción: 29/05/2018 | Aceptado: 17/04/2019 \\ Correspondencia a través de ORCID: Kyoko Ito-Morales \\ 0000-0001-9095-8797 \\ Citar: Ito-Morales, K (2019). Estado actual y desafío del uso de TIC en la enseñanza de japonés \\ como lengua extranjera. ReiDoCrea - Monográfico sobre Perspectivas transnacionales en la \\ enseñanza de lenguas, 8(3), 141-157.
}

Resumen: El desarrollo de las herramientas TIC facilita el acceso a la información y materiales necesarios a los usuarios de las TIC. Los estudiantes interesados en el aprendizaje de una nueva lengua pueden acercarse tanto a la lengua como a la cultura, por simple acción de, por ejemplo, cliquear los videos de YouTube, visitar páginas webs, tomar cursos gratuitos online, etc.

El profesorado de lenguas puede estar satisfecho al tener estudiantes muy motivados con los conocimientos previos facilitados por el uso de TIC. Al mismo tiempo, pueden estar confusos por la múltiple variedad de información. Los estudiantes también puede que se encuentren que lo que han aprendido por su cuenta con la ayuda de las TIC, no está del todo de acuerdo con lo que se enseña en clase.

Este es el caso frecuentemente observado en las clases de japonés como lengua extranjera. Gracias al boom del manga y el anime, el número de estudiantes que desean aprender japonés en las universidades españolas está gradualmente creciendo cada año. No obstante, el japonés, especialmente para los alumnos occidentales, es una de las lenguas más complejas y difíciles de aprender, debido a su carácter ideográfico, su gramática, la variedad de expresiones verbales y no verbales de cortesía, etc. De hecho, los tonos y formas de expresar cortesía se estructuran dentro de la cultura comunitarista o grupista, y se enseñan de múltiples formas o a veces incorrectamente en los materiales TIC ofrecidos. Además, los manuales utilizados en las aulas simplifican o ignoran este asunto.

¿Cómo se puede solventar este vacío entre estos materiales para enseñar japonés correctamente? Este trabajo tiene como principal propósito analizar diversos materiales TIC y de manuales de enseñanza, así como buscar posibles herramientas para aplicar en las clases de japonés y mejorar su adquisición.

Palabras clave: Lengua extranjera. Tecnología educacional.

\section{Current situation and challenge of the use of ICT in teaching Japanese as a foreign language}

\begin{abstract}
The development of ICT tools facilitates to access to needed information and materials for ICT users. Students interested in learning a new language can approach both the language and the culture, by simple action of, for example, clicking YouTube videos, visiting websites, taking free online courses, etc. Language teachers can be satisfied by having students who are highly motivated by the prior knowledge provided by the use of ICT. At the same time, they may be confused by the vast variety of information. Students may also find that what they have learned on their own with the help of ICT is not entirely in accordance with what is taught in class.

This is the case frequently observed in classes of Japanese as a foreign language. Thanks to the boom of manga and anime, the number of students who want to learn Japanese in Spanish universities is gradually growing each year. However, Japanese, especially for Western pupils, is one of the most complex and difficult languages to learn, due to its ideographic character, the grammar, the variety of verbal and nonverbal expressions of courtesy, etc. In fact, the tones and ways of expressing courtesy are formed within the community or group-oriented culture. Therefore sometimes those are taught incorrectly by the ICT materials. In addition, the manuals used in classrooms simplify or ignore this issue.

How can you fill this gap between these materials to teach Japanese correctly? The main purpose of this work is to analyze various ICT materials and teaching manuals, as well as to look for possible tools to apply in Japanese classes to improve the acquisition.
\end{abstract}

Keywords: Foreign languages. Educational technology.

\section{Introducción}

El desarrollo actual de las herramientas TIC es llamativo. Gracias a él, se facilita el acceso a la información y a materiales necesarios e interesantes por su manejo rápido 
y cómodo. Si se enfocan a los estudiantes interesados en aprender una nueva lengua extranjera, se pueden acercar tanto a la lengua como a la cultura, por la simple acción de, por ejemplo, cliquear los videos de YouTube, visitar páginas webs, tomar cursos gratuitos online, etc. A saber, los alumnos de lenguas extranjeras tienen ahora más opciones y métodos de estudios para elegir, de lo que había antes de la aparición de herramientas TIC. Con este fenómeno, por un lado, el profesorado puede estar satisfecho al tener estudiantes muy motivados con conocimientos previos. Sin embargo, por otro lado, tanto estudiantes como profesores pueden estar confundidos por las múltiples variedades de información, metodología, explicación e interpretación sobre la lengua estudiada y su cultura. Aparentemente, es indispensable por parte de los alumnos, saber por dónde y cómo navegar en la oleada de webs existente, y por parte de los profesores, conocer el mar en el que están sus alumnos, y saber dirigirlos para que lleguen a su destino: la adquisición correcta de la lengua extranjera.

La confusión causada por la sobrecarga de información es un caso frecuentemente observado en las clases de japonés como lengua extranjera. Gracias al boom del manga y el anime, mundialmente el número de estudiantes de japonés está aumentando (Fukunaga, 2006, p.207). España no es una excepción ya que el número de estudiantes que desean aprender japonés en las universidades españolas está gradualmente creciendo cada año. Se observa que un cierto porcentaje de los alumnos nuevos de japonés tienen un conocimiento previo sobre dicha lengua por haber estado estudiándola autodidácticamente. Además, como son de la generación digital con habilidad de manejo de nuevas tecnologías, vienen preparados con materiales facilitados por las TIC. De hecho, estos materiales accesibles contribuyen al aumento del interés para estudiar japonés (Fukunaga, 2006, p.207). No obstante, el japonés, especialmente para los alumnos occidentales, es una de las lenguas más complejas y difíciles de aprender, debido a su carácter ideográfico, su gramática, la variedad de expresiones verbales y no verbales de cortesía, etc. Por ejemplo, el Departamento de Estado de EE.UU. categoriza el japonés, junto con el chino, el coreano y el árabe, como una de las lenguas más difícil de aprender (Noda, 2018, p.4; U.S. Department of State, n.d.). Además de la dificultad de la lengua japonesa en sí, se observa que la gran cantidad de materiales ofrecidos por TIC son incontrolables para asegurar calidad y fiabilidad de la enseñanza. Desgraciadamente, eso causa confusión para los estudiantes de japonés.

Igualmente, en el caso del japonés en España es oportuno examinar la influencia de las herramientas TIC sobre el aprendizaje de lenguas extranjeras, porque en comparación con otros países occidentales como EE.UU. y países de Europa, existen muy pocas instituciones oficiales que enseñan el japonés como lengua extranjera, y no se enseña el japonés en niveles inferiores a la universidad (Japan Foundation, 2017). Dada esta situación así, los aficionados del japonés van a elegir el camino autodidáctico favorecido por las TIC, y algunos llegarán a matricularse en las clases universitarias de japonés. Es decir, las aulas universitarias son donde se encuentran por primera vez el alumnado autodidacta conducido por las TIC y el profesorado con métodos tradicionales de enseñanza de japonés. Naturalmente, no es sorprendente de que los estudiantes se encuentren confundidos por el gap de su conocimiento basado en las informaciones web, así como los profesores asombrados por el nivel (a veces muy alto) de japonés de la clase inicial, pero al mismo tiempo confundidos por conocimientos parciales o tendenciosos de ellos.

¿Cómo se puede solventar este vacío entre la enseñanza de japonés disponible con TIC y el aula, para que los estudiantes puedan utilizar los métodos que sientan más cómodos y aprendan japonés para poder ejercerlo de verdad? Este trabajo tiene como principal propósito analizar diversos materiales TIC de la enseñanza de japonés, así como buscar las posibles herramientas para aplicar en las clases de japonés y mejorar su adquisición.

\section{Marco teórico}


A pesar de algunas preocupaciones sobre la bajada de la alfabetización a causa de la inserción rápida de la tecnología, como expresa Sanders (1994), Collins y Blot piensan que la alfabetización nunca ha sido sólo para poder leer y escribir, sino que es algo más amplio que incluye otras formas como la transmisión oral o escrita por imágenes, y conceptos variados como textos, lenguas, significados, tecnología, cultura, poder, identidad etc. (Collins y Blot, 2003; Fukunaga, 2006, p.207). Además, estamos en la era de las multiliteracies porque las nuevas tecnologías están siendo suplementarias de las modalidades tradicionales de alfabetización (Collins y Blot, 2003, p.168). Igualmente ellos argumentan que, para contribuir a la alfabetización tecnológica, el simple acto de "click" al acceder a internet o para navegar sin objetivo no significa nada, sino que es necesario que un individuo sea consciente de su acción y su intención porque es una parte de la adquisición y síntesis de información expansiva y acertada (Collins y Blot, 2003, pp.171-172).

Gee sitúa las nuevas tecnologías como una llave potencial para los jóvenes para sobrevivir en la sociedad moderna. Basado en la teoría de multiliteracies, Gee argumenta que la práctica de la alfabetización (aunque en este caso el término de alfabetización incluye múltiples sentidos reflexionados recientemente) ha sido, y sigue siendo algo esencial para poder tener éxito económica y sociopolíticamente en la sociedad (Gee, 2004, p.91). Según él, el lenguaje académico que se enseña en la escuela y que distingue a las personas inteligentes y educadas, es una parte de la alfabetización antigua (old literacies). Como consecuencia, alcanzar el lenguaje académico ya no es un factor suficiente para la supervivencia en la sociedad actual (Gee, 2004, p.94). Gee sigue afirmando que la sociedad en la que vivimos está en la fase de un nuevo capitalismo, donde ser eficiente no es el requisito fundamental para el éxito, sino adquirir conocimiento sobre las nuevas tecnologías y habilidad de trabajar en equipo. Para poder reaccionar rápidamente ante los factores cambiantes que rodean la sociedad, como el desarrollo y las innovaciones científicas, la globalización del mercado, las migraciones de personas, etc., hacen falta esas competencias modernas. Por eso, lo que importa y realmente es útil son las habilidades, logros y experiencias previas de TIC y colaborar con otras personas; en palabras de Gee, "portfolio" (2004, p.97). De este modo, los jóvenes, o en términos de Gee, los millennials deben equiparse con diversas habilidades, logros y experiencias que se pueden adquirir dentro y fuera de colegio, además de a través de productos de alta tecnología (Gee, 2004, pp.107-108). Aunque Gee no da mucha importancia de la enseñanza en las aulas, el profesorado puede enfocar su clase para contribuir a la necesidad de los jóvenes de la sociedad moderna con alta tecnología. Una manera sería introducir materiales on-line y utilizar las herramientas TIC. Los aspectos positivos de la introducción de herramientas TIC en las clases de lenguas extranjeras son aumento de motivación, eficiencia, apoyo a la autonomía en el aprendizaje, flexibilidad espacial y temporal, entre otros (Arón-Macià, 2012; Kikuchi, 2008; Cooke-Plagwitz, 2008). Por estos motivos, hoy en día los profesores no pueden evitar estar al tanto del desarrollo tecnológico para poder responder a la demanda de los estudiantes, sobre todo los jóvenes de la generación digital (Arón-Macià, 2012, p.99; Kikuchi, 2008, p.44). A veces, por razones como las infraestructuras, restricciones de horario y plan de estudio, o escasez de personal, no se dispone de posibilidades del uso de herramientas TIC en el aula. Aun así, de todas maneras, los alumnos jóvenes de la generación digital nativa acuden a los materiales on-line como parte de sus portfolios. En este caso, los profesores modernos tienen un nuevo cargo de mentor para dirigir y aconsejar a los estudiantes (Kikuchi, 2008, p.55). Por lo tanto, para el profesorado actual de lenguas extranjeras, ya es un requisito inevitable manejar las herramientas básicas TIC y conocer mínimamente los materiales on-line, para apoyar la educación del alumnado de la generación digital.

Llegados a este punto, hemos fundamentado la necesidad de la introducción de herramientas TIC en la enseñanza de las lenguas extranjeras. A continuación, estudiamos la situación de la lengua japonesa como lengua extranjera y aclaramos la importancia del manejo de materiales TIC en el caso del japonés. 


\section{Estado actual del japonés como lengua extranjera Información General}

El japonés es la lengua que se habla básicamente sólo en Japón, y cuenta con unos 122 millones de personas que la hablan como su lengua materna (Knowlton, 2014). Por el tamaño relativamente pequeño de la población de japonés hablante, se puede considerar como una de las lenguas minoritarias.

El japonés es una de las lenguas más difíciles de aprender. Según el departamento del estado de los EE.UU. (US. Department of State, n.d.), el japonés se categoriza como "super-hard language", uno de las cinco lenguas más difíciles de aprender para los angloparlantes, que requieren unas 88 semanas o 2.200 horas de clases para llegar al nivel de competencia profesional de trabajo (S3) (equivalente al C1 del MCER) (SkyLearn, n.d.), mientras lenguas de la categoría I (danés, francés, italiano, portugués y español entre otros) necesitan unas 24-30 semanas, de la categoría II (alemán, indonesio, swahili, etc.) exigen aproximadamente 36 semanas y de la categoría III (suomi, búlgaro, hindú, checo, turco, etc.) demandan unas 44 semanas.

Aun así, se observa un incremento de personas que estudian japonés, especialmente desde los años 80 entre la gente de negocios por motivo del boom económico japonés, y del de los años 90 entre los jóvenes gracias al boom de manga y anime (Okuda y Okuda, 2017, p.8; Noda, 2018, p.13). The Japan Foundation informa que en el año 2015 hubo unos 3.650.000 estudiantes de japonés en todo el mundo, con 16.179 instituciones de enseñanza y 64.108 profesores (Japan Foundation, 2017a, p.7). Se observa en la figura 1, un ligero descenso del número de alumnos entre 2012 y 2015 . Sin embargo, Japan Foundation y otros investigadores explican que esa bajada fue causada por el aumento de alumnos autodidactas que son cada vez más jóvenes y son de la generación digital, por lo tanto, suelen estudiar por sí mismos utilizando internet y otras herramientas TIC (Japan Foundation, 2017, p.9; Okuda y Okuda, 2017, p.50; Fukunaga, 2006, p.206).

Fig.1. Número de alumnos registrados en instituciones

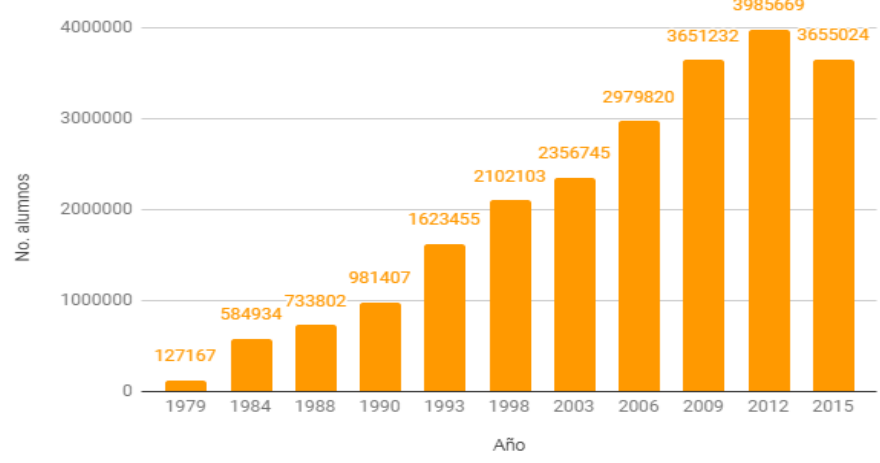

(Fuente: Japan Foundation, 2017a, p.8)

En el nivel europeo, igualmente se observa un aumento de la enseñanza de japonés en casi todos los países, causado principalmente por el boom de la cultura popular. En el caso de Francia, además de la cultura popular, mantiene interés por los estudios sobre Japón y su tradición, y eso favorece el crecimiento de instituciones superiores de enseñanza de japonés (Japan Foundation, 2017a, p.37). Es importante mencionar que, entre los países con más número de alumnos de japonés, España es el único país entre los cinco países más activos de la enseñanza de japonés, en el que no se enseña japonés en el nivel de la enseñanza primaria, secundaria y bachillerato, mientras Inglaterra, Francia y Alemania ofrecen clases de japonés desde las escuelas primarias e Italia desde las secundarias (Japan Foundation, 2017a, pp.36-37). Debido a la falta de enseñanza de japonés en la educación obligatoria, en España se observa un gran 
número de alumnos autodidactas por interés sobre el manga, anime, los videojuegos, etc. (Abe, 2009, p.31).

Fig.2. Número de instituciones, profesores y alumnos en Europa

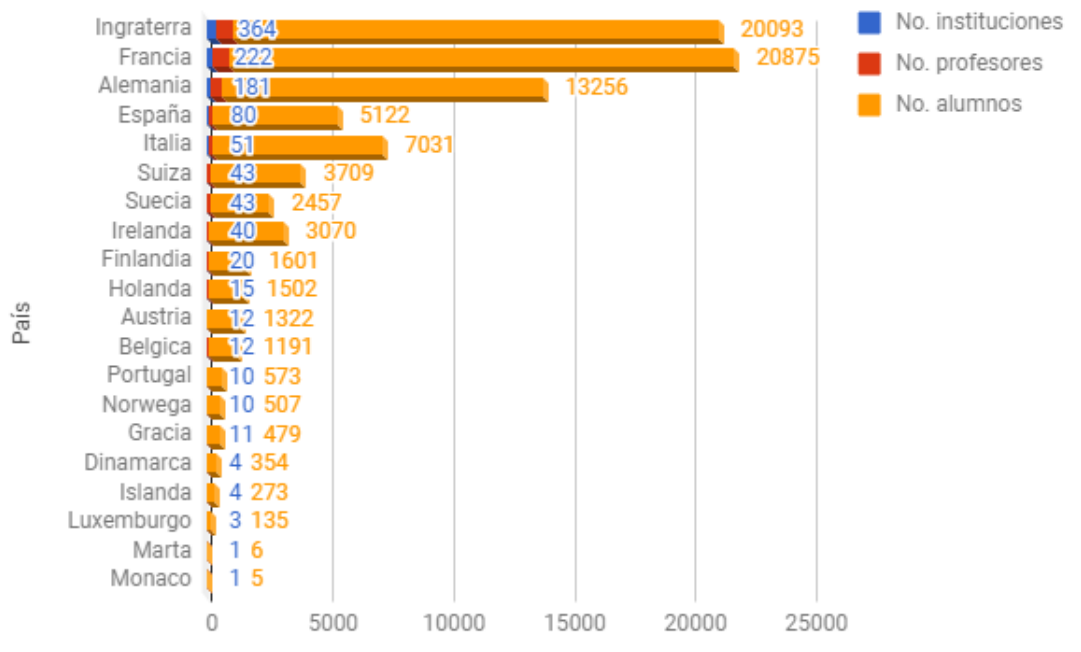

(Fuente: Japan Foundation, 2017a, p.36, elaboración propia)

\section{Cursos universitarios en España}

La enseñanza de japonés estructurada en España comenzó en 1967, cuando abrieron dos tipos de cursos de japonés: uno para los investigadores de ciencias y otro para los trabajadores de hostelería y turismo. Posteriormente, la Asociación de Estudios Orientales y la Embajada de Japón en España empezaron cursos para el público general en 1971, pero apenas duraron un año por razones económicas. No obstante, las Escuelas Oficiales de Idiomas que son públicas en España, comenzaron cursos de japonés en los años setenta. La primera fue en Barcelona en el año 1971 y la siguiente en Madrid en 1975. En la actualidad, aproximadamente cuarenta escuelas oficiales de toda España están impartiendo cursos de japonés (Embajada de Japón en España, 2016). Como hemos visto anteriormente, hasta ahora España no tiene cursos de japonés ofrecidos en la educación primaria, secundaria y bachillerato. En cuanto a las universidades españolas, se abrió un curso en la Universidad Autónoma de Madrid en 1985, al principio con la ayuda de la Japan Foundation. La universidad más avanzada en la enseñanza de japonés es la Universidad Autónoma de Barcelona que tiene experiencia desde 1988. Al principio, los cursos de ambas universidades fueron de lengua minor (la segunda o tercera lengua extranjera). No obstante, en 2003, el gobierno español creó un nuevo grado de estudios Asia-orientales (una carrera de dos años), que abrió la posibilidad de la inclusión del japonés en su currículo. Además, la Autónoma de Barcelona instaló el grado de estudios japoneses como una carrera de cuatro años desde 2009. Actualmente, existen unas veinte universidades que ofrecen grados con la posibilidad de escoger japonés como sus estudios principales (Embajada de Japón en España, 2016), y es por eso por lo que tiene importancia y esperanza en desarrollar y establecer la enseñanza de japonés en España (Abe, 2009, p.31).

\section{Asignaturas en la Universidad de Granada}

En el caso de la universidad de Granada (UGR), donde la autora imparte sus clases, hay dos posibilidades de matricularse para estudiar japonés: en el Centro de Lenguas Modernas (CLM), o en la Facultad de Filosofía y Letras (FFL). Como los cursos en el CLM no se reconocen como cursos universitarios, los alumnos que desean el 
reconocimiento de estudios sobre japonés toman asignaturas impartidas en la FFL. En la FFL, la lengua japonesa es tratada como una de las lenguas minor, y se ofrece a los alumnos de varios grados como el Grado en Lenguas Modernas y sus Literaturas, el grado en Literatura Comparada, los grados de Estudios Árabes, Ingleses, Franceses, Hispánicos, etc. Actualmente, hay tres niveles: Japonés Inicial, Intermedio y Avanzado. Todas las asignaturas son de 6 créditos, 2 clases semanales y 2 horas cada clase. Cuentan con unos 70 alumnos en el nivel inicial, 40 en el intermedio y 10 en el avanzado. Además, hay un nivel superior en el Máster oficial en Estudios de Asia Oriental.

Debido a la oferta de japonés para amplios grados, además de un considerable número de estudiantes autodidactas (aproximadamente un tercio en la clase inicial), se observa una gran diferencia del nivel de interés, motivación y conocimiento previo de la lengua japonesa en todas las asignaturas de japonés. No obstante, prácticamente todos los alumnos mencionan un contacto previo con la cultura popular japonesa a través de anime y manga, esencialmente transmitidos por programas de televisión desde su infancia. Finalmente, pocos, pero hay algunos estudiantes con experiencia de tomar cursos de japonés en algunas academias.

\section{Dificultades de los alumnos}

En general, los alumnos de japonés expresan unas dificultades comunes. Primero, como se ha presentado anteriormente, para los hablantes de lenguas europeas, la lengua japonesa en sí es difícil de aprender. Segundo, por ser una de las lenguas minor, el número y las horas de clases son muy limitadas para aprender una lengua tan distinta del español. Tercero, frecuentemente los alumnos aficionados al manga $y$ anime enfrentan una confusión considerable entre el japonés que se enseña en la clase y el otro tipo de japonés que encuentran en los cómics y las películas. Como consecuencia, a menudo los estudiantes manifiestan sus frustraciones por no poder notar el progreso de aprendizaje (sobre todo cuando se comparan con el de otras lenguas extranjeras europeas), y un tipo de desmotivación o desvinculación por no poder mantener sus intereses en japonés provocado por la cultura popular japonesa.

Cuando se enfocan las distintas competencias, se perciben particularmente dos temas con mayor dificultad de aprendizaje: la cortesía y los kanji.

Controlar el nivel de cortesía, es decir mantener la distancia personal y elegir el lenguaje de cortesía adecuado en cada situación es vital en la sociedad japonesa que sigue siendo muy tradicional, jerárquica y comunitarista. Los tonos y formas de expresar cortesía se estructuran dentro de la cultura comunitarista o grupista (Fukunaga, 2006, p.218). Los nativos japoneses continuamente toman decisiones de utilizar japonés en estilo formal o informal, para marcar las relaciones de uchi/soto (dentro y fuera) de sus conexiones interpersonales (Iwasaki, 2011). A pesar de su importancia, curiosamente la gran mayoría de libros de texto de la enseñanza de japonés como lengua extranjera no enseñan el tema de la cortesía desde el principio. Además, cuando lo enseñan en el nivel intermedio, las descripciones y explicaciones son muy simplificadas dándosele demasiada importancia al estilo formal (Iwasaki, 2011, p.71). Existen varias interpretaciones de la falta de enseñanza de cortesía. Rounds, Flasgraf y Seya opinan que la cortesía como una de las culturas implícitas, no se enseña de la misma manera que asignaturas de historia o literatura, sino que se aprende a través de las interacciones sociales en varios contextos socioculturales (1997, p.28; Iwasaki, 2011; Tomita, 2012). Para que pueda reproducirse el ambiente sociocultural japonés en el aula, Neustupunỳ piensa que hace falta tres relaciones distintas: relaciones de alumno-profesor, alumnoalumno y alumno-visitante (1977, p.143). Como es muy complicado conseguir la relación de alumno-visitante en el aula, las clases de japonés solían ignorar el tema de cortesía. Algunos estudiantes acaban aprendiendo las reglas de cortesía japonesa sólo una vez que están en Japón viviendo y tras pasar un tiempo mal y confundido en el contexto real de la sociedad japonesa. Además, Iwasaki observa que los japoneses no esperan que los no-nativos controlen la cortesía japonesa, por lo tanto, los profesores tampoco dan 
mucha importancia a introducir la variedad de cortesía en la clase (Iwasaki, 2011, p.73). Tal vez hasta hace unos años, esta estrategia (aparentemente muy irresponsable) no causaba muchos problemas, porque los que consiguen experimentar la cortesía yendo a Japón eran minoritaria. No obstante, actualmente, el desarrollo de las TIC permite que la gente experimente mucho más la cultura japonesa sin salir de su habitación de ordenador. Los mismos manga, anime, cine o música por YouTube transmiten el lenguaje vivo y actual de la sociedad japonesa, incluyendo la gran variedad de cortesía. Y eso causa confusión al contrastar la lengua enseñada en los textos y clases. Por ello, ya no se debe ignorar el tema de la cortesía en la clase, sino que hay que intentar buscar la manera de explicar el tema integralmente. Así, los profesores deben saber las fuentes de información a las que acceden los alumnos y poder entender las confusiones que ellos sufren.

La otra dificultad es aprender kanji. Kanji es uno de los tipos de caracteres que utiliza el japonés. Son originalmente de China, pero adaptado al japonés. Como uso diario, deben poder manejarse unos $2.000 \mathrm{kanji}$, aunque en las clases de japonés en la UGR, estudiamos aproximadamente 100 kanji en el nivel inicial, 300 en intermedio y 600 en avanzado. De hecho, los nativos japoneses tardan toda la educación obligatoria de nueve años y unos más de educación superior para poder aprender los kanji de uso diario. Es por ello por lo que es muy comprensible la dificultad de los alumnos de japonés (Aoki, 2012, p.132; Yamashita y Maru, 2000). No sólo por la cantidad, sino por la característica de los kanji de ser de ideológicos o logográficos, aumenta la dificultad de aprendizaje entre estudiantes españoles y de lengua-europea hablantes, también porque sus lenguas maternas no contribuyen al proceso de aprendizaje (Aoki, 2012, p.132). Sin embargo, a menudo los profesores nativos japoneses no comprenden la dificultad de sus alumnos. Por eso hace falta desarrollar otras estrategias de aprendizaje (Matsumoto, 2013; Yamashita y Maru, 2000). Aun así, actualmente, el método tradicional de memorización (rote-learning) sigue siendo más utilizado y aparentemente más eficiente para los estudiantes de lenguas-europeas hablantes, igual que los nativos japoneses (Mori, 2014, p.416). Para salir del aprendizaje monotónico, puede que las herramientas TIC sirvan para contribuir el desarrollo de materiales más creativos. Por otro lado, no todos los alumnos vienen con el conocimiento nulo de kanji; los alumnos chinos (mayoritariamente del programa de intercambio) y los alumnos españoles con estudios previos de chino tienen ventajas e inconvenientes. Como ventaja, alrededor del $70 \%$ de kanji en japonés y en chino son de la misma forma, por lo cual se facilita la memorización (Aoki, 2013, p.132). No obstante, a pesar de ser las mismas escrituras, los significados y maneras de leer son distintos al chino, por lo que causa una mayor confusión entre estos alumnos. Reconocer las dificultades y necesidades de cada alumno debe ser considerado a la hora de la búsqueda de materiales TIC también.

\section{Análisis de materiales TIC}

El aumento de e-learning, o aún más allá, el m-learning (que utiliza los materiales online accesibles por móviles) (Munday, 2016, p.84), es un reflejo del aumento de materiales on-line. De hecho, el número de materiales on-line y aplicaciones para el aprendizaje de japonés está incrementándose cada vez más. A continuación, se presentarán unos ejemplos de materiales TIC para el aprendizaje de japonés como lengua extranjera.

\section{Materiales generales}

En general, los materiales TIC de japonés como lengua extranjera no han sido muy innovadores. Se observa una inconformidad de estudiantes de japonés, sobre todo los jóvenes contemporáneos que están acostumbrados a estudiar utilizando aplicaciones de internet y teléfono móvil, por falta los materiales TIC (Noda, 2018, p.16). Una de las 
razones principales del tardío comienzo y el desarrollo lento en este campo fue el problema de la codificación de las letras japonesas (Kato, 2016, p.115). Actualmente, ya el tema de codificación se ha superado bastante, y el docente de japonés está intentando ponerse al día en materiales TIC de aprendizaje de lenguas. Al mismo tiempo, las versiones de japonés de las aplicaciones del aprendizaje de lenguas como Duolingo están saliendo al mercado poco a poco, a pesar de las dificultades causadas por algunas particularidades del japonés como la cortesía y la cantidad de letras (Hagiwara, 2017).

Además de ser gratis, Duolingo (https://www.duolingo.com/) ofrece cursos de lenguas bastante integrales. Se basa en el sistema de space-out practice, interleaving y favorece el desarrollo de competencias variadas (Munday, 2016, p.85). Sin embargo, como desventajas, Duolingo ofrece el curso de japonés sólo para angloparlantes. Además, el contenido cubre sólo hasta el nivel CEFR (Common European Framework of Reference for Languages) A1 o N1 del JLPT (Japanese Language Proficiency Test) (Hagiwara, 2017), por lo que aparenta ser apto para los alumnos de nivel inicial.

Fig. 3. LingoDeer

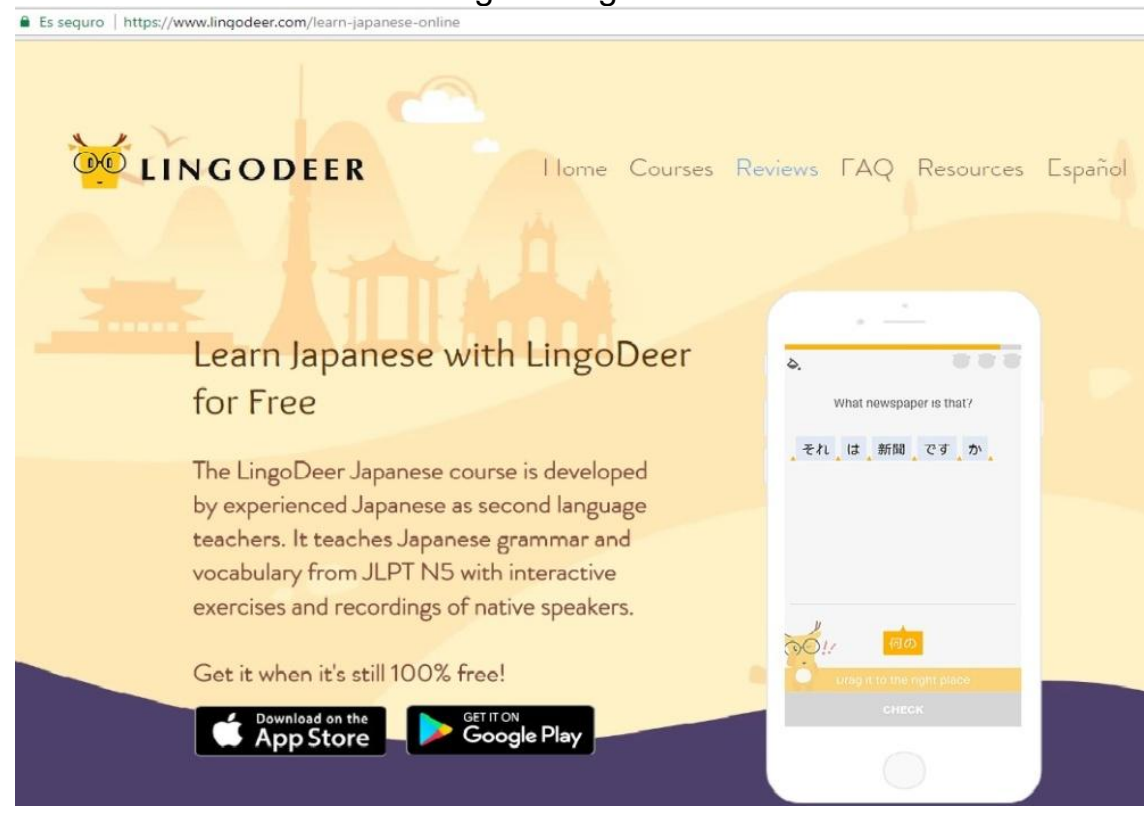

Parecido a Duolingo, LingoDeer (https://www.lingodeer.com/) ofrece cursos de idiomas, pero esta aplicación se especializa en las lenguas asiáticas. LingoDeer supera a Duolingo por la posibilidad del aprendizaje de japonés para hispano hablante (https://www.lingodeer.com/español) y el aumento del nivel (hasta N3). Se observan algunas mejoras más que Duolingo como la introducción de flashcards. Por lo tanto, consideramos que puede servir al aprendizaje de los alumnos españoles de japonés.

Existen algunos materiales desarrollados por los japoneses nativos también. Nihonmaru (http://www.nihonmura.net/), y U-biq (http://study.u-biq.org/english.html) son unos de los ejemplos y ofrecen varios ejercicios gramáticos y escrituras japonesas. Sin embargo, los materiales TIC producidos por los japoneses nativos a menudo presuponen la capacidad de los estudiantes de poder escribir en japonés en ordenadores, aunque en realidad la gran mayoría no tiene por qué saber escribir en japonés. Por lo tanto, este tipo de materiales requiere una habilidad TIC específica para japonés.

Fig. 4. Nihonmura 


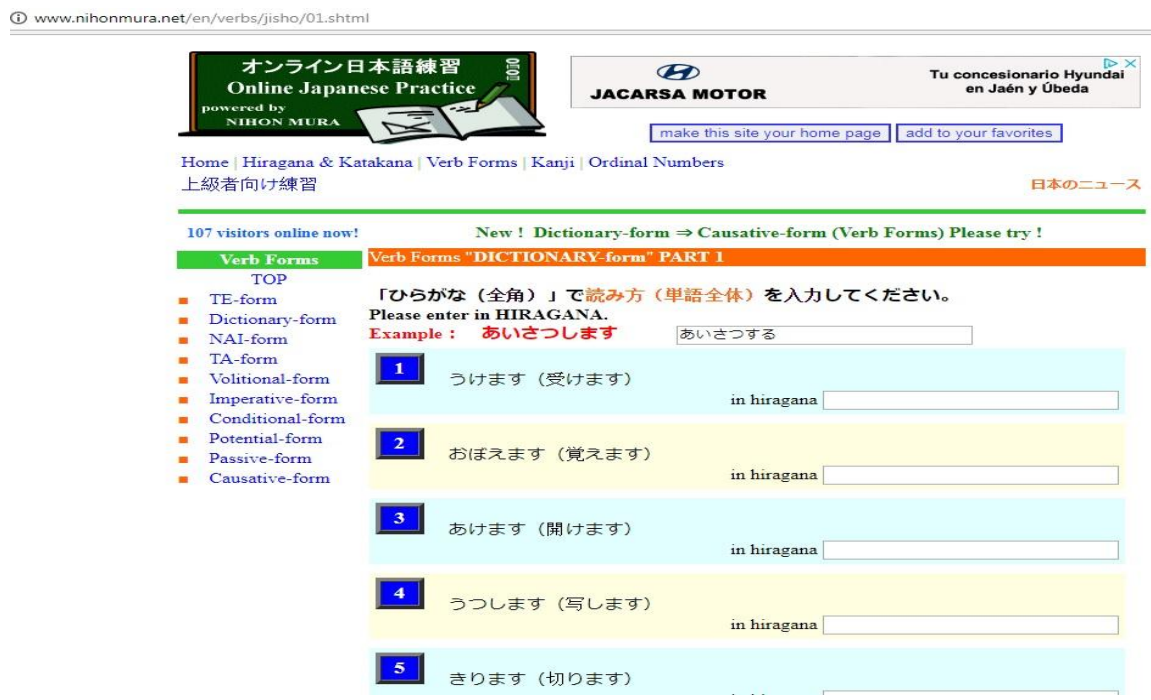

Se observan varias aplicaciones que apoyan al aprendizaje basado en la repetición. Memorise (https://www.memrise.com/), Quizlet (https://quizlet.com/es), Anki (https://apps.ankiweb.net/), etc. ofrecen plataformas para que los alumnos creen sus fichas de estudios, o que utilicen los que ya hayan sido creados por otros. Algunos, como Quizlet, tienen más variedades de ejercicios y se observa un gran potencial de jugabilidad.

Lang-8 (http://lang-8.com/) facilita un espacio gratis para que los usuarios intercambien los servicios de corrección de sus escritos por los nativos. Es una nueva forma de aprender a escribir sin necesidad de corrección por parte de profesores. No obstante, aunque son nativos, los que corrigen los escritos en Lang-8 no son profesionales de enseñanza de las lenguas extranjeras. Por ello, puede que sea insuficiente para aprender las causas de los errores gramaticales.

Por último, hay varias páginas que ofrecen, no sólo ejercicios sino también cursos de japonés gratuitos on-line. Tea Kim (http://www.guidetojapanese.org/learn/), Imabi (http://www.imabi.net/), PuniPuni (http://www.punipunijapan.com/), y Bunpro (https://www.bunpro.jp/) cubren una amplia temática para aprender japonés en inglés. Kira-teaching (http://www.kira-teachings.com/) ofrece cursos y ejercicios en español vía varios medios como su página web, quizlet, youtube, etc. Como algunos cursos de Kirateaching están basados en el texto "Minna no Nihongo" (el más utilizado en las universidades españolas), algunos de mis alumnos acuden a esta página cuando estudian autónomamente. A pesar de que es una herramienta excelente para apoyar la autonomía de los estudiantes, se ha observado en diversas ocasiones confusión de los estudiantes al conocer distintas explicaciones en Kira-teaching y en las aulas. En este sentido, acercarse a lo que dicen estas páginas por la parte del profesorado de japonés ayudaría a solucionar las dudas y confusiones provocadas por los usos de herramientas TIC.

Como hemos visto, existen una gran variedad de páginas y aplicaciones que facilitan la autodidáctica de los alumnos de japonés. No obstante, se perciben mejoras necesarias para favorecer a los estudiantes, especialmente a los alumnos españoles en este caso. Primero, la falta de materiales TIC en español es grave. Aunque algunos estudiantes no tienen miedo de aprender japonés a través del inglés, hay otros que no se sienten capacitados o cómodos con las explicaciones en inglés. Segundo, a pesar de que un considerable número de estudiantes llegan a aprender japonés motivados por sus gustos por el manga y el anime, salvo Japanese in Anime \& Manga por Japan Foundation (http://anime-manga.jp/), no se encuentra prácticamente ningún material utilizado o motivado por manga y anime. La razón más importante -en nuestra opiniónes el tema de los derechos de autor (Kato, 2016, pp.84-90) que restringen el uso de 
dichas obras. Aun así, es una pena no poder vincular el boom del manga con materiales TIC para el aprendizaje de japonés. Tercero, pese a que la oferta de materiales on-line es abundante, no hay muchas herramientas que trabajen para desarrollar las habilidades de forma integral. Concretamente, la gran mayoría de herramientas analizadas aquí no promueven suficientemente el desarrollo de la habilidad comunicativa. Por último, cuanto más nivel, se encuentran menos materiales. Los materiales para el nivel inicial son muchos, e incluso excesivos. Sin embargo, para el nivel intermedio ya hay menos, y para el nivel avanzado o superior existen muy pocos.

Fig. 5. Japanese in Anime \& Manga

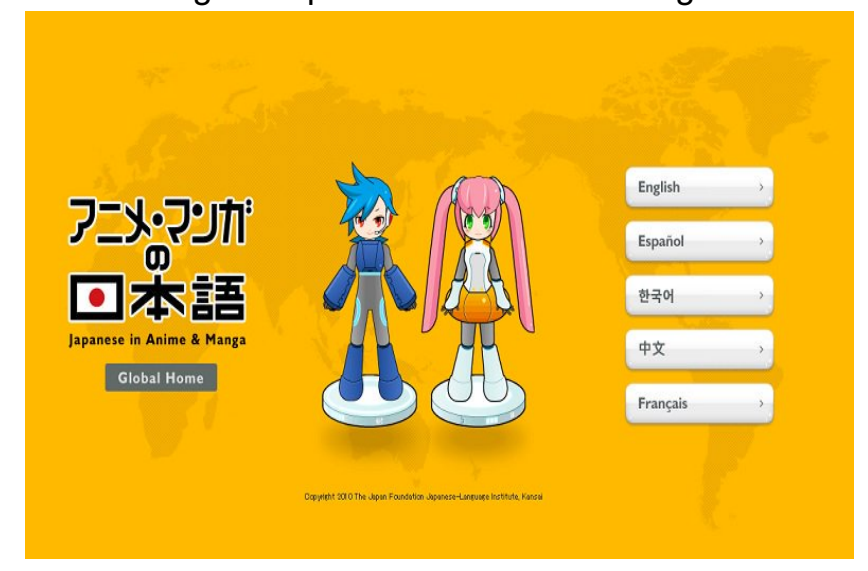

Para cubrir esa falta de materiales en los niveles superiores, parece oportuno considerar el uso de materiales on-line para niños japoneses nativos. Por ejemplo, los periódicos para niños que están escritos en japonés fácil, pueden ser útiles para los alumnos del nivel intermedio o más, además de poder familiarizarse con las noticias en Japón. Las páginas de cuentos infantiles (por ejemplo http://www.e-hon.jp/) incluyen ocasionalmente cuentos y leyendas antiguas de Japón que sirven para acercarse a la literatura japonesa. Escuchar a las noticias on-line para niños puede complementar los ejercicios auditivos, y los materiales preparados para colegios como NHK for School (http://www.nhk.or.jp/school/) facilitan el entendimiento por los efectos audiovisuales. Es cierto que algunos alumnos no quieren materiales demasiado infantiles. Aun así, creemos que la selección correcta de materiales para niños nativos puede servir mucho para conocer bien el entorno general y educativo de Japón y desarrollar las habilidades por vías diferentes.

Fig. 6. News Web EASY

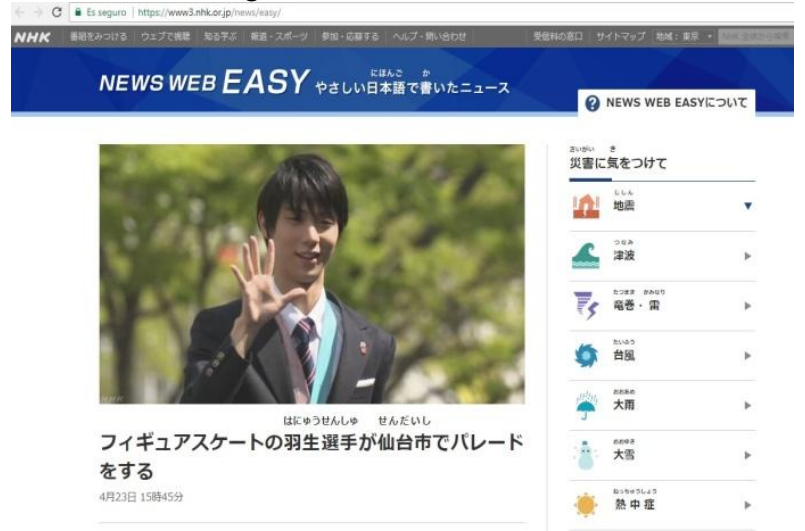




\section{Materiales para la enseñanza de cortesía}

En la sección anterior, se han analizado las dificultades de aprendizaje de japonés, especialmente se ha detallado la dificultad del manejo de cortesía y kanji. Por ese motivo, examinaremos someramente los materiales TIC que puedan servir para superar esas dificultades.

Con relación al tema de la complejidad de la cortesía japonesa, es excesivamente difícil encontrar materiales on-line que ayuden al estudio. Ya que el tema de cortesía se introduce en la clase de intermedio-avanzado normalmente, y la escasez de materiales para niveles altos se ha observado anteriormente, no hay material que apoye a un mejor entendimiento de la cortesía. Además, recordemos la idea de Neustupunỳ de tres relaciones necesarias para el aprendizaje de la cortesía, a saber, relaciones de alumnoprofesor, alumno-alumno y alumno-visitante (1977, p.143), donde en un entorno autodidacta no se cumplen ninguna de esas relaciones humanas. En algunos casos como ver documentales que reflejan bien la jerarquía de la sociedad japonesa, puede que ayuden a la adquisición del concepto de cortesía japonesa, pero requerirá un nivel suficientemente alto de japonés para entender los diálogos y observar las distancias entre la gente y sus usos de cortesía. Por otro lado, los alumnos interesados en manga y anime siguen viendo los programas sin darse cuenta de la diferenciación y adecuación del uso de la cortesía. Es por ello por lo que, para el profesorado, hace falta conocer bien los materiales a los que los alumnos acceden para familiarizarse con Japón y el japonés fuera de clase. Asimismo, es indispensable estar preparado para añadir explicaciones para que los estudiantes estén sensibilizados a diferenciar los usos y variedades corteses.

Un posible acercamiento en el futuro al aprendizaje de la cortesía utilizando las herramientas TIC es la introducción de los mundos virtuales. Por ejemplo, Second Life hace posible crear otra sociedad en un entorno virtual, y ya existe una comunidad japonesa actualmente. No obstante, llevar a los estudiantes de japonés allí para experimentar el uso de la cortesía es demasiado irreal, puesto que la gran mayoría de usuarios japoneses de Second Life son jóvenes de 20-39 años (Wada, 2010, p.109) y no representan la jerarquía de la sociedad japonesa real. No obstante, no debe negarse la utilidad y posibilidad del mundo real completamente, porque, por otro lado, se observa una tendencia de comunitarismo japonés en Second Life (Wada, 2010, p.107). Un tour con estudiantes guiado por el profesor puede que dé una experiencia de sentir la comunidad japonesa y que sirva para aprender el uso de cortesía sin viajar realmente a Japón.

\section{Materiales para la enseñanza de kanji}

La otra dificultad observada entre los estudiantes de japonés es aprender una cantidad considerablemente grande de kanji. Al contrario de la cortesía, las herramientas TIC para ayudar al aprendizaje de kanji es abundante.

WaniKani (https://www.wanikani.com/) es básicamente fichas de kanji, pero adopta el método de spaced repetition. Para aprender cada kanji con sus maneras de leer y significados, WaniKani ofrece suficientes ejercicios. Asimismo, CosCom (https://www.coscom.co.jp/japanesekanji/) facilita ejercicios con la opción de escuchar sonido. Las desventajas de estas herramientas son, uno, servicio ofrecido sólo en inglés, y dos, sólo los primeros niveles son gratis. Otras herramientas presentadas anteriormente como LingoDeer, Nihonmura, Anki, Quizlet, etc. incluyen la sección de aprender kanji. No se percibe mucha diferencia entre uno y otro, y como siempre, a más niveles menos materiales. 


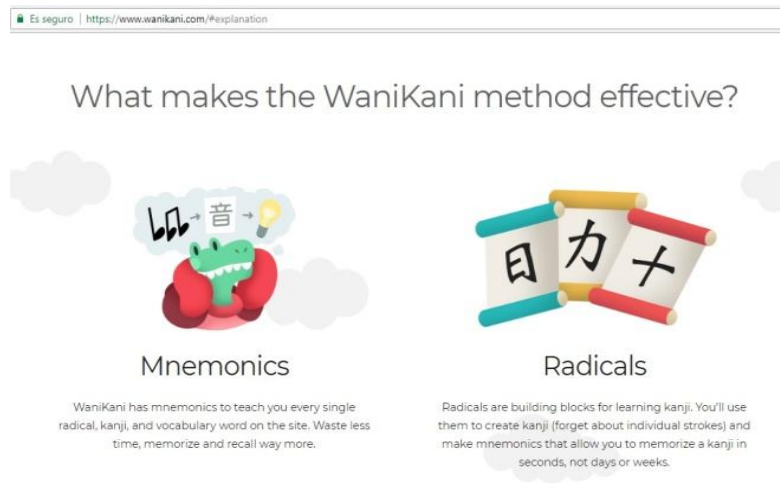

La sección de kanji de Japanese in Anime \& Manga deja margen de jugabilidad para evitar la repetición monótona en el aprendizaje, aunque los kanji que ofrecen son demasiado específicos a los escenarios de anime y manga, por lo cual no son muy útiles para los entornos habituales de vida de los estudiantes universitarios.

Por último, hay materiales para niños japoneses nativos que pueden ser útiles para aprender kanji. Ōtotsu shōgakkō (http://www.ipt.bb4u.ne.jp/ g-benkyo/) es uno de los ejemplos que también muestra la posibilidad de jugar y aprender. La cuestión es el nivel y orden de introducción de kanji, ya que normalmente el sistema de introducción de kanji para japoneses nativos y para los alumnos de japonés es distinto según la utilidad de cada kanji. Por lo tanto, hay que buscar el nivel que corresponde a cada alumno.

Fig. 8. Ōtotsu shōgakkō

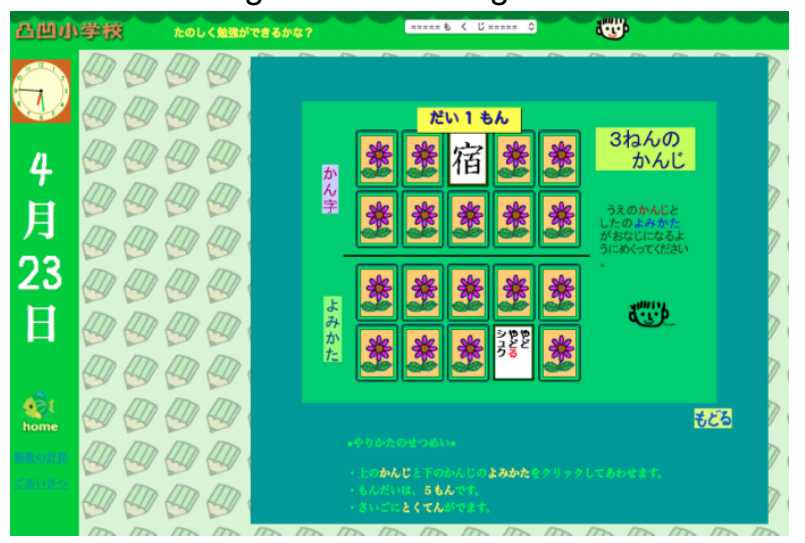

En resumen, al contrario de la cortesía, hay bastantes materiales para apoyar el aprendizaje de kanji. Sin embargo, se nota la falta de opciones en español y la mayoría está sólo en inglés. De igual modo, la falta de materiales (sobre todo gratis) para los niveles altos es notable en el caso de herramientas para los kanji. Además, se contempla que la gran mayoría de aplicaciones y páginas web proponen kanji y sus significados descontextualizados, sin considerar los contextos y entornos donde aparecen esos kanji. La falta de esos contextos es grave para los alumnos de japonés, porque en la clase la gran mayoría de estudiantes acuden a los profesores para saber la selección apropiada de kanji en un contexto particular. Por ello, es importante que el profesorado tenga en cuenta este asunto. Por último, se sigue la búsqueda de herramientas prácticas para los alumnos chinos o alumnos de chino. En este caso, requiere extender la investigación a los materiales ofrecidos en chino.

\section{Desafío del uso en aula}


Hemos analizado algunos materiales TIC para el aprendizaje de japonés como lengua extranjera. La gran mayoría de las herramientas revisadas están diseñadas para apoyar la autonomía en el aprendizaje de los alumnos. Ahora bien, ¿sería beneficioso utilizar algunos materiales TIC en el aula?

Tal y como hemos estudiado, los alumnos, especialmente los de la generación digital que son los alumnos actuales de las universidades españolas, usan las herramientas TIC diariamente para varios objetivos incluyendo sus estudios. Ya la alfabetización no significa poder leer y escribir. Siguiendo la teoría de Multiliteracies de Collins y Blot (2003), la alfabetización de la era moderna incluye poder leer y manejar textos, tecnología, cultura popular, etc. Además, como dice Gee, estos "jóvenes mileniales" tienen que saber vivir con las nuevas tecnologías para poder tener éxito en la sociedad moderna (2004, p.99). Y el profesorado también debe estar al tanto de la tendencia actual y satisfacer la demanda del alumnado. Por lo tanto, se debe considerar la inclusión de materiales TIC en el aula de lenguas extranjeras.

La introducción de materiales on-line puede contribuir a algunas ventajas. Primero, puede motivar a los alumnos por la novedad en la clase. En el caso de la clase de japonés es evidente, puesto que se tiende a adoptar el método tradicional de lectura y traducción de textos. De hecho, desde la experiencia de la autora, se puede observar un cambio radical de la motivación de los estudiantes cuando se introducen algunos ejercicios diferentes con el apoyo de herramientas TIC. Así pues, se puede contribuir a la dinámica de la clase. Segundo, las herramientas TIC pueden aportar a la eficacia de la clase. En efecto, hemos estudiado la realidad de las clases de japonés, particularmente en la UGR, donde el japonés es lengua minoritaria con una limitación seria del número de horas, excesivos alumnos en algunas clases, y gran diferencia del nivel de interés, motivación y conocimientos previos de la lengua japonesa. En esta situación, el uso de herramientas TIC facilita la atención individualizada a la necesidad de cada alumno. Por ejemplo, a un estudiante con la dificultad del aprendizaje de kanji, se le pueden recomendar algunos materiales introducidos en este trabajo para que vaya estudiando. Otro alumno con problemas del uso de partículas se le puede dirigir a los materiales on-line de ejercicios dramáticos. De esta manera, las horas en el aula pueden dedicarse a actividades más culturales, comunicativas y cooperativas para realizar tareas que sólo pueden desarrollar en la clase. Tercero, algunos materiales TIC puede facilitar la labor de los profesores, concretamente la elaboración de materiales de ejercicios y repartirlos. Por una parte, hay muchos materiales elaborados y accesibles on-line para complementar los ejercicios dentro y fuera de clase. Por otro lado, aplicaciones como Quizlet, Memorise y Anki nos pueden servir para crear materiales propios para que sean más adecuados y concretos para la clase. De esta manera, la distribución de materiales es sólo dar la dirección de web, es más económico y eficiente, y los alumnos pueden estudiar donde y cuando quieran. En definitiva, la introducción de herramientas TIC nos puede traer múltiples ventajas.

Sin embargo, no se pueden negar algunos factores negativos. Se observa a veces la limitación en la instalación de aparatos tecnológicos, tanto en las aulas universitarias (por ejemplo, restricción del acceso a internet, falta de actualización de ordenadores), como los ordenadores de los estudiantes. Además, desde la revisión general de materiales hecha en las secciones anteriores, hemos aprendido que hay que tener en cuenta el tema de los derechos de autor, sobre todo de materiales relacionados con manga y anime. Hay que evaluar previamente antes de recomendar a los alumnos la calidad y fiabilidad de los materiales para poder dirigir correctamente el uso de esos materiales. Asimismo, se sabe que hay falta de materiales para los niveles avanzados en el caso del japonés. Puede cubrirse ese inconveniente al utilizar materiales para japoneses nativos, pero en este caso, hay que facilitar el ajuste a la lengua japonesa. Hemos descubierto que la gran mayoría de materiales on-line sirven para desarrollar una habilidad específica (gramática, vocabulario, kanji, etc.). Por lo tanto, debe trabajarse bien en el aula para el desarrollo de otras competencias, particularmente la habilidad comunicativa que es difícil de ser llevada a cabo con herramientas TIC para 
compensar ese hueco. Y, por último, hay que reconocer que no todos los estudiantes están dispuestos a la introducción de herramientas TIC en la clase de lengua. No todos los alumnos, sea de la generación digital o no, tienen la misma capacidad del manejo de herramientas TIC, porque se supone que los jóvenes tienen la capacidad básica del empleo de esas herramientas, pero generalmente (o por lo menos en el caso de la UGR) esa competencia no está enseñada formalmente en la educación regularizada, no sólo por motivo de la capacidad personal, sino también por razón de las infraestructuras, es decir, no todos los alumnos tienen aparatos electrónicos necesarios para el uso de materiales on-line.

Considerando los aspectos positivos y negativos de la adaptación de las herramientas TIC en el aula, en el caso de la clase de japonés como lengua extranjera, de momento parece sensato el uso de tales herramientas TIC como una parte del "blended learning" (Fukada, 2018, p.2). De este modo, se puede corresponder a la demanda de los alumnos de la generación digital, se puede contribuir a la dinámica y eficiencia de la clase, al mismo tiempo que no diseñar la clase con una dependencia total a las TIC, puede cubrir los aspectos negativos mencionados más arriba. Sin embargo, el uso de herramientas TIC por parte del alumnado debe siempre ir acompañado por la diligencia, recomendación y corrección del profesorado para evitar confusiones y apoyar la adquisición apropiada de la lengua japonesa.

\section{Conclusión}

En este trabajo, se han revisado varios materiales TIC para el aprendizaje de japonés como lengua extranjera. Tomamos el caso de la enseñanza de japonés en España que muestra sus particularidades, concretamente por un lado el aumento notable de los interesados en el japonés motivados por manga y anime, y por el otro lado la falta de instituciones de enseñanza en los niveles inferiores a la universidad. Esa situación conduce a los aficionados de japonés al método autodidacta al uso de herramientas TIC. Es por ello por lo que es importante que los profesores de japonés conozcan los materiales disponibles on-line y las herramientas TIC más apropiadas.

Desde el análisis de materiales TIC, hemos descubierto que hay varias páginas web y aplicaciones interesantes para el aprendizaje de japonés en general y de kanji. Sin embargo, la gran mayoría está en inglés y hay muy pocos en español, hay escasez de materiales para los niveles altos, y esas herramientas no son ideales para el desarrollo de la competencia comunicativa del japonés. Además, se ha estudiado que no hay prácticamente ningún material on-line que apoye al aprendizaje del control del nivel de cortesía, que es un factor indispensable para entender la cultura japonesa y la gran mayoría de los estudiantes expresa la dificultad de aprenderla.

Considerando estos puntos, se concluye que la adaptación de herramientas TIC como una parte del blended learning parece adecuada para la enseñanza de japonés en las universidades españolas actualmente. La aplicación ocasional y adicional de herramientas TIC tanto en aula como fuera de aula puede contribuir a la demanda y la motivación del alumnado, además de a la dinámica y la eficiencia de la clase. No obstante, la dependencia total de los materiales TIC pone en riesgo la calidad de la clase por razones como la falta de infraestructuras, los materiales pertinentes para el desarrollo de la competencia integral del manejo del japonés, y la insuficiencia de habilidad para la tecnología por la parte de los estudiantes y los profesores. En todo caso, en el mundo contemporáneo actual con alumnos de generación digital, es sólido e importante reconocer que los profesores de lenguas extranjeras deben adquirir la habilidad del manejo esencial de las herramientas TIC, conocer mínimamente los materiales básicos on-line, y poder dirigir y aconsejar a los estudiantes sobre esos materiales TIC. La inclusión de herramientas TIC nos permitirá acercarnos a la lengua japonesa desde múltiples ángulos, relacionando más temas alrededor de la lengua japonesa y su cultura, con la ayuda del uso de varios sentidos (vista, oído, etc.), para que el proceso de adquisición de la lengua sea más auténtico, natural y cómodo, 
además de ser divertido y motivador. Por ello, se espera que la investigación sobre el uso de herramientas TIC para la enseñanza de lenguas extranjeras continúe activa y creativa.

\section{Referencias}

Abe, S. (2009). スペイン・マドリードの大学における日本語学習者の言語学習ビリー フ (Japanese language learners' beliefs about language learning surveyed in university in Madrid, Spain) (en japonés). Journal of School of Foreign Languages, Nagoya University of Foreign Studies 37, 25-62.

Aoki, N. (2012). The challenge of motivation: Teaching Japanese kanji characters to students from diverse language backgrounds. En X. Song y K. Cadman (Eds.), Bridging Transcultural Divides: Asian Languages and Cultures in Global Higher Education, (pp.131-153). Adelaide, Australia: University of Adelaide Press.

Arón-Macià, E. (2012). The role of technology in teaching languages for specific purposes courses. The Modern Language Journal, 96, 89-104. doi: 10.1111/i.1540-4781.2120.01299.x

Collins, J. y Blot, R. K. (2003). Literacy and literacies: Text, power and identity. Cambridge, New York, Melbourne, Madrid, Cape Town, Singapore y San Paulo: Cambridge University Press.

Cooke-Plagwitz, J. (2008). New directions in CALL: An objective introduction to Second Life CALICO Journal, 25 (3), 547-557. Recuperado de http://www.jstor.org/stable/calicojournal.25.3.547

Embajada de Japón (2016). Estudiar en Japón. Recuperado de http://www.es.embjapan.go.jp/itpr_es/00_000021.html

Fukuda, J. (2018). 日本語教育におけるテクノロジー (Tecnología en la enseñanza de japonés) (en japonés). Recuperado de https://www.aatj.org/resources/publications/book/Technology_Fukada.pdf

Fukunaga, N. (2006). Those anime students: foreign language literacy development through Japanese popular culture. Journal of Adolescent and Adult Literacy, 50 (3), 206-222. doi:10.1598/JAAL.50.3.5

Gee, J. P. (2004). Shape-shifting portfolio people. En J. P. Gee, Situated language and learning: A critique of traditional schooling, (pp. 91-115). New York y Londres: Routledge.

Hagiwara, M. (2017). How we invented a new way to teach one of the most difficult languages to learn. Recuperado de http://making.duolingo.com/how-weinvented-a-new-way-to-teach-one-of-the-most-difficult-languages-to-learn

Iwasaki, N. (2011). Learning L2 Japanese "politeness" and "impoliteness": Young American men's dilemmas during study abroad. Japanese Language and Literature, 45 (1), 67-106. Recuperado del http://www.jstor.org/stable/41151381

Japan Foundation (2017a). Survey report on Japanese-language education abroad 2015. 
http://www.jpf.go.jp/j/project/japanese/survey/result/dl/survey_2015/Report_all_ e.pdf

Japan Foundation (2017b). 日本語教育 国 地域別情報 スペイン（２０１７７）(El estado de la enseñanza de japonés en España) (en japonés). Recuperado de https://www.jpf.go.jp/j/project/japanese/survey/area/country/2017/spain.html\#JI SSHI

Kato, K. (2016). 視聴覚メディアと日本語教育 (Los medios de comunicación audiovisuales y la docencia de japonés). Tokio: ALC Press Inc.

Kikuchi, S. (2008). モバイル学習としての iTunesU と Second Life の可能性 (La potencialidad de iTunesU y Second Life para el aprendizaje de lenguas móviles. Journal of School of Foreign Languages, Nagoya University of Foreign Studies, 34, 37-61. Recuperado de http://www3.nufs.ac.jp/ kikuchi/2008-nufs.pdf

Knowlton, E (27 mayo 2014). The easiest and most difficult languages for English speakers to learn. Business Insider. Recuperado de http://www.businessinsider.com/the-hardest-languages-to-learn-2014-5

Kubota, R. (2018). 日本語教育における文化 (Cultura japonesa en la enseñanza de japonés) (en japonés). Recuperado de https://www.aatj.org/resources/publications/book/Culture_Kubota.pdf

Matsumoto, K. (2013). Kanji recognition by second language learners: Exploring effects of first language writing system and second language exposure. The Modern Language Journal 97(1), 161-177. doi: 10.1111/j.1540-4781.2013.01426.x

Mori, Y. (2014). Review of recent research on kanji processing, learning, and instruction. Japanese Language and Literature, 48 (2), 403-430. Recuperado de http://www.jstor.org/stable/24394416

Munday, P. (2016). The case for using DUOLINGO as part of the language classroom experience. La Revista Iberoamericana de la Educación Digital, 19 (1), 83-101. doi: 10.5944/ried.19.1.14581

Nazikian, F. y Park, J. (2016). How to develop "21st century skills" in foreign language education. Japanese Language and Literature, 50 (2), 347-373. Recuperado de http://www.jstor.org/stable/24892016

Neustupnỳ, J. V. (1977). Some strategies for teaching Japanese honorifics. The Journal of the Association of Teachers of Japanese, 12 (2 / 3), 135-147. doi: $10.2307 / 489163$

Noda, M. (2018). アメリカの高等教育機関における日本語教育(El estado de enseñanza de japonés en las instituciones superiores en EE.UU.) (en japonés). Recuperado de https://www.aatj.org/resources/publications/book/HigherEd_Noda.pdf

Okuda, K. y Okuda, H. (2017). 日本語教育の現状 (El estado de la enseñanza de japonés) (en japonés), Tokio: ALC Press. 
Rose, H. (2013). L2 learners' attitudes toward, and use of, mnemonic strategies when learning Japanese kanji. The Modern Language Journal, 97 (4), 981-992. doi: 10.1111/j.1540-4781.2013.12040.x

Rounds, P. L., Falsgraf, C. y Seya, R. (1997). Acquisition of sociolinguistic competence in a Japanese immersion school. The Journal of The Association of Teachers of Japanese, 30 (2), 25-51. doi: 10.2307/489657

Sanders, B. (1994). A is for ox: Violence, electronic media, and the silencing of the written word. California: Pantheon Books.

SkyLearn (n.d.). Cuadro de equivalencias/certificado. Recuperado de http://www.spain.skylearn.cn/index.php/portfolio/cuadro-de-equivalenciascertificado

Tomita, A. (2011). Personal growth through intercultural communication: Engaging native speakers and reflecting learning in Japanese language curriculum. En X. Song y K. Cadman (Eds.), Bridging Transcultural Divides: Asian Languages and Cultures in Global Higher Education, (155-188). Adelaide: University of Adelaide Press. doi: 10.20851/j.ctt1sq5w6k.12

U.S. Department of State (n.d.). FSI's experience with language learning. Recuperado de https://www.state.gov/m/fsi/sls/c78549.htm

Wada, T. (2010). 日本における仮想空間「セカンドライフ」の利用実態 (El estado actual del uso del espacio virtual "Second Life" en Japón). Geographical Science, 65 (2), 104-126. doi: 10.20630/chirikagaku.65.2_104

Yamashita, H. y Maru, Y. (2000). Compositional Features of Kanji for Effective Instruction. The Journal of The Association of Teachers of Japanese, 34 (2), 159178. doi: $10.2307 / 489552$ 